\title{
Modeling Opponents in Adversarial Risk Analysis
}

\author{
David Rios Insua, ${ }^{1}$ David Banks, ${ }^{2}$ and Jesus Rios ${ }^{3, *}$
}

\begin{abstract}
Adversarial risk analysis has been introduced as a framework to deal with risks derived from intentional actions of adversaries. The analysis supports one of the decisionmakers, who must forecast the actions of the other agents. Typically, this forecast must take account of random consequences resulting from the set of selected actions. The solution requires one to model the behavior of the opponents, which entails strategic thinking. The supported agent may face different kinds of opponents, who may use different rationality paradigms, for example, the opponent may behave randomly, or seek a Nash equilibrium, or perform level- $k$ thinking, or use mirroring, or employ prospect theory, among many other possibilities. We describe the appropriate analysis for these situations, and also show how to model the uncertainty about the rationality paradigm used by the opponent through a Bayesian model averaging approach, enabling a fully decision-theoretic solution. We also show how as we observe an opponent's decision behavior, this approach allows learning about the validity of each of the rationality models used to predict his decision by computing the models' (posterior) probabilities, which can be understood as a measure of their validity. We focus on simultaneous decision making by two agents.
\end{abstract}

KEY WORDS: Adversarial risk analysis; Bayesian model averaging; decision analysis; opponent modeling; simultaneous games

\section{INTRODUCTION}

Recent applications in counterterrorism, cybersecurity, and competitive corporate decision making have driven renewed interest in developing practical tools and theory for analyzing the strategic calculation of intelligent opponents who must act in scenarios with random outcomes. We use the term adversarial risk analysis (ARA) to describe approaches in which the solution is based upon an explicit Bayesian model of the capabilities, probabilities, and utilities used by the opponent in his

${ }^{1}$ Institute of Mathematical Sciences, ICMAT-CSIC, Madrid, Spain.

${ }^{2}$ Department of Statistical Sciences, Duke University, Durham, NC, USA.

${ }^{3}$ IBM T.J. Watson Research Center, Yorktown Heights, NY, USA.

*Address correspondence to Jesus Rios, IBM T.J. Watson Research Center, 1101 Kitchawan Rd. Yorktown Heights, NY 10598, USA; jriosal@us.ibm.com analysis. See Refs. 1-6 for various concepts, methods, and applications.

In ARA, the aim is to support one of the players who will use a decision-analytic approach to solve her decision-making problem. To this end, she needs to forecast the actions of the other agents and, based on her own choice, the outcomes that she and her opponents will receive. This is not a new solution concept; it can be viewed as a Bayesian approach to game theory, and was proposed, nonconstructively, by Kadane and Larkey ${ }^{(7)}$ and Raiffa. ${ }^{(8)}$ The approach has been criticized by Harsanyi ${ }^{(10)}$ and Myerson, ${ }^{(11)}$ among others. From a practical standpoint, the main obstacle in implementing the decision-analytic approach has been the lack of explicit mechanisms that allow the supported decisionmaker to encode her subjective probabilities about the components in her opponents' decision making. ARA deals with this problem within the framework of a Bayesian model for the supported decisionmaker's uncertainty. She may 
face various kinds of opponents, who may use different rationality paradigms. However, she herself is a rational expected utility maximizer, in accordance with the Bayesian decision theory developed by Savage. ${ }^{(12)}$

Specifically, this article treats opponents who may act at random, or be Nash equilibria seeking, level- $k$ thinking, mirror equilibria seeking, or prospect maximizing, but other kinds are possible. We describe how to model each of these opponents, and then use Bayesian model averaging to incorporate uncertainty about the rationality paradigm used by the opponent. Details on Bayesian model averaging can be found in Refs. 13 and 14; a longer treatment is given in Ref. 15. As discussed, this increases the set of models that may be used and enables a fully decision-theoretic solution that properly reflects the supported analyst's uncertainty.

The structure of the article is as follows. We first present the problem of two-person discrete simultaneous games, and briefly compare the game-theoretic and ARA approaches. Next we describe models for the various kinds of rationality that may be used by opponents, and then how those models can be combined to reflect uncertainty about such kind of rationality. We also examine the cognitive burden of the analyses that pertain to different rationality paradigms. To illustrate the methodology, we use examples of simple games relevant to counterterrorism, adapted from previous work in this area.

\section{BASICS}

The two-person discrete simultaneous game is described by the multiagent influence diagram in Fig. 1. These diagrams were proposed by Koller and Milch, ${ }^{(16)}$ and use rectangles to indicate decisions, ovals to indicate random variables, and hexagons to indicate preferences.

This structure includes such games as auctions, the Prisoner's Dilemma, and other classic examples. But it also includes the important simultaneous Defend-Attack family of games, discussed by many authors, including Refs. 17-21. For counterterrorism applications, there are strong advantages to the ARA perspective since it partitions the modeling into standard risk analyses conditional on the decisions that are taken by the opponents, and strategic considerations, which depend upon the utilities and personal probabilities of the opponents, as well as their solution concepts. ${ }^{(22)}$

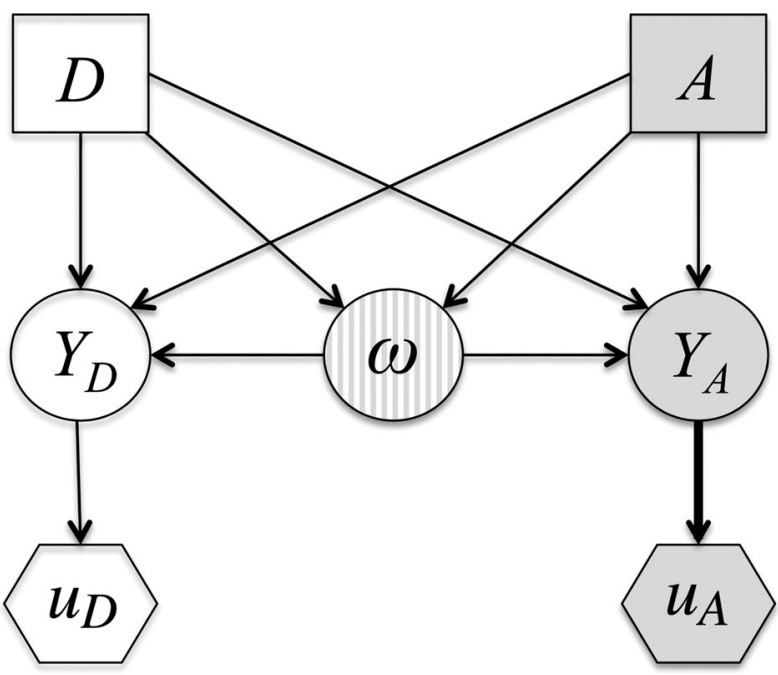

Fig. 1. A two-person simultaneous game.

In our discussion, there are two agents, Daphne and Apollo. Daphne chooses from a finite set of actions $\mathcal{D}=\left\{d_{1}, \ldots, d_{m}\right\}$, while Apollo simultaneously chooses from the finite set $\mathcal{A}=\left\{a_{1}, \ldots, a_{n}\right\}$. These choices correspond to the rectangles indicated by the corresponding initial. Daphne and Apollo receive payoffs $Y_{D}$ and $Y_{A}$, respectively, which depend upon both of their actions $(d, a)$ and (in general) a random outcome $\omega$; for example, in a Prisoner's Dilemma game, this randomness might correspond to the chance of getting a strict or lenient judge. The hexagons in the diagram indicate the utilities $u_{D}$ and $u_{A}$ received by Daphne and Apollo, respectively, from the payoffs $Y_{D}$ and $Y_{A}$.

Daphne's expected utility associated with the pair of actions $(d, a) \in \mathcal{D} \times \mathcal{A}$ is:

$$
\psi_{D}(d, a)=\int u_{D}(d, a, \omega) p_{D}(\omega \mid d, a) d \omega,
$$

where $u_{D}(d, a, \omega)$ represents the utility she gets from a payoff $Y_{D}(d, a, \omega)$ and $p_{D}(\omega \mid d, a)$ represents her beliefs about the chance of obtaining the outcome $\omega$, given the chosen pair of actions $d$ and $a$. Similarly, Apollo's expected utility is:

$$
\psi_{A}(d, a)=\int u_{A}(d, a, \omega) p_{A}(\omega \mid d, a) d \omega .
$$

Under the assumption of common knowledge, ${ }^{(11,23)}$ Daphne and Apollo are expected utility maximizers who know the other's probabilities, utilities, and choice set, and both know that all of this is commonly known. In that case, the game may be described in 
bimatrix form:

$$
d\left(\begin{array}{c}
a \\
\vdots \\
\cdots \psi_{D}(d, a), \psi_{A}(d, a)
\end{array}\right) .
$$

When the common knowledge assumption holds, players can predict with certitude their opponent's best responses to each of their actions by solving the opponent's decision problem. Apollo's best response, known by Daphne, is then:

$$
R_{A}(d)=\operatorname{argmax}_{a \in \mathcal{A}} \psi_{A}(d, a),
$$

and, similarly, Daphne's best response, known by Apollo, is:

$$
R_{D}(a)=\operatorname{argmax}_{d \in \mathcal{D}} \psi_{D}(d, a) .
$$

These predictive models of best response $R_{A}$ and $R_{D}$ are used to find a pure-strategy Nash equilibrium solution for the game, consisting of a pair of actions $\left(d^{*}, a^{*}\right)$ that are best responses to each other, so that $d^{*}=R_{D}\left(a^{*}\right)$ and $a^{*}=R_{A}\left(d^{*}\right)$. Since a Nash equilibrium solution is not guaranteed to exist for discrete games, often the action sets are extended to include randomized strategies, consisting of probability distributions over the original set of actions, so that a solution can always be found for the extended game. ${ }^{(24)}$ However, the common knowledge assumption is implausible in many applications. See Refs. 9, 25 , and 26, among others, for detailed discussions of this issue. ARA avoids this problematic assumption through Bayesian modeling.

Specifically, Daphne must acknowledge her uncertainty about how her opponent solves his decision problem. Depending upon the kind of rationality paradigm she believes Apollo uses, she may have to place subjective distributions over the utilities and probabilities held by Apollo. She may also have to model what Apollo thinks about her decision behavior. Things can quickly become complicated. To start with a simple example, suppose Daphne believes that Apollo has some value v(a) associated with each of his possible actions, and that he will select the value maximizing action $a^{*}=$ $\operatorname{argmax}_{a \in \mathcal{A}} v(a)$. This model would predict Apollo's action if Daphne knew the values $v\left(a_{i}\right)=v_{i}$, for $i=1, \ldots, n$. But without telepathy, Daphne must proceed as a Bayesian and describe her uncertainty about $\left(v_{1}, \ldots, v_{n}\right)$ through a joint distribution $\left(V_{1}, \ldots, V_{n}\right) \sim F$, where $F$ represents her probabilistic beliefs about the values Apollo holds for his alternatives. Thus, Daphne believes that Apollo will choose action $a_{i}$ with probability $p_{D}\left(a_{i}\right)=\mathbb{P}_{F}\left(V_{i}\right.$ is the largest member of $\left.\left\{V_{1}, \ldots, V_{n}\right\}\right)$. Now, in order to maximize her own expected utility, Daphne solves

$$
\max _{d} \psi_{D}(d)=\sum_{i=1}^{n} \psi_{D}\left(d, a_{i}\right) p_{D}\left(a_{i}\right)
$$

to find the action $d$ that maximizes her expected utility. The point of this simple example is to show how Daphne can replace common knowledge with subjective belief, and then proceed to select the action that is optimal under Bayesian decision theory.

The next section extends this simple example by showing how Daphne can obtain the probabilities $p_{D}(a)$ that she needs for her solution under various models for the kind of rationality that Apollo may use. At various points, we shall mention random probabilities and utilities, which, when invoked, will refer to a common probability space ${ }^{(27)}(\Theta, \mathcal{F}, \mathcal{P})$ with atomic elements $\theta$ (i.e., single events in the sample space).

\section{PROBABILISTIC MODELS OF OPPONENT BEHAVIOR}

In general, Daphne will not know what kind of rationality (or solution concept) Apollo uses in choosing his decision. There are many kinds of rationality described in the literature, and this section shows how Daphne should use ARA with respect to five of them. Later, we combine them into a mixture model, so that Daphne can incorporate her personal uncertainty about Apollo's solution concept, both to make her immediate decision and learn about Apollo's rationality, if there is repeated play. To illustrate the ideas, we use examples of the simultaneous Defend-Attack game.

\subsection{Nonstrategic Opponents}

First, assume that Daphne believes that Apollo is nonstrategic. In that case, it is as if she were playing Nature, in the standard decision-theoretic parlance, ${ }^{(28)}$ since Apollo makes his decision at random, without regard to Daphne's action. Based on past data and/or expert opinion, Daphne will selfelicit her distribution $p_{D}(a)$.

If the contest is repeated and Apollo lacks memory of previous moves, then he chooses randomly and independently each time. Then, Daphne would learn her distribution over his action space through a multinomial distribution, for which a Dirichlet 
prior would be the usual choice. Here, Daphne initially assumes a Dirichlet distribution $\left(p_{1}, \ldots, p_{n}\right) \sim$ $\mathcal{D}\left(\alpha_{1}, \ldots, \alpha_{n}\right)$, where $p_{i}$ is her initial subjective probability that Apollo chooses action $a_{i}$. Then, after $T$ iterations of the game, suppose Apollo has selected action $a_{i}$ exactly $h_{i}$ times, so that the counts for his actions are $\left(h_{1}, \ldots, h_{n}\right)$, with $\sum_{i} h_{i}=T$. Bayesian inference shows that Daphne's posterior distribution for Apollo's choice is $\mathcal{D}\left(\alpha_{1}+h_{1}, \ldots, \alpha_{n}+h_{n}\right)$, whose density function shall be designated $f(p \mid h)$.

Daphne can now evaluate each $d \in \mathcal{D}$ by computing her corresponding expected utilities as follows:

$$
\begin{aligned}
\psi_{D}(d)= & \int\left[\sum_{i=1}^{n} \psi_{D}\left(d, a_{i}\right) p_{i}\right] f(p \mid h) d p \\
& =\sum_{i=1}^{n} \psi_{D}\left(d, a_{i}\right) E_{p \mid h}\left[p_{i} \mid \text { data }\right]
\end{aligned}
$$

Then, taking

$$
p_{D}^{N S}\left(a_{i}\right)=E_{p \mid h}\left[p_{i} \mid \mathrm{data}\right]=\frac{h_{i}+\alpha_{i}}{T+\sum \alpha_{i}},
$$

for $i=1, \ldots, n$, as point probability forecasts for Apollo's nonstrategic choice, Daphne's maximum expected utility action can be obtained by solving:

$$
d_{N S}^{*}=\operatorname{argmax}_{d} \sum_{i=1}^{n} \psi_{D}\left(d, a_{i}\right) p_{D}^{N S}\left(a_{i}\right),
$$

which determines her best choice in the nonstrategic case.

In a slightly more general vein, suppose that Apollo has memory, and can recall the previous $T$ games. For example, suppose Daphne and Apollo are playing Rock-Paper-Scissors. A memoryless Apollo would choose at random (possibly with unequal probabilities). If Apollo has memory of the previous $T$ outcomes, but he is not strategic, then his probability for selecting, say, Rock in the next game will depend on the number of times he has played it the last $T$ plays and had Rock win. So the randomness of his play conditions on the past.

To simplify the exegesis, we assume he can recall only the preceding game, and we also limit the outcome of that game to discrete levels indexed by $\omega \in \Omega$, the set of possible outcomes. These outcomes indicate the degree of success from his action. Both of these simplifying assumptions can be easily weakened. In this case, through the Markov property,
Daphne can use a matrix beta prior, as in Ref. 29, to learn the corresponding parameters through

$$
\left(p_{1}, \ldots, p_{n}\right) \mid d_{i}, a_{j}, \omega \sim \mathcal{D}\left(\alpha_{1}^{i j \omega}, \ldots, \alpha_{n}^{i j \omega}\right) .
$$

If after a $\left(d_{i}, a_{j}, \omega\right)$ game Apollo has selected action $a_{k}$ exactly $h_{k}^{i j \omega}$ times, then

$$
\begin{array}{r}
\left(p_{1}, \ldots, p_{n}\right) \mid d_{i}, a_{j}, \omega, \text { data } \sim \mathcal{D}\left(\alpha_{1}^{i j \omega}+h_{1}^{i j \omega},\right. \\
\left.\ldots, \alpha_{n}^{i j \omega}+h_{n}^{i j \omega}\right),
\end{array}
$$

and Daphne could use the probability mass function

$$
\begin{gathered}
p_{D}^{N S}\left(a_{k} \mid d_{i}, a_{j}, \omega, \text { data }\right)=E\left[p_{k} \mid d_{i}, a_{j}, \omega, \text { data }\right] \\
=\frac{\alpha_{k}^{i j \omega}+h_{k}^{i j \omega}}{A^{i j \omega}+T^{i j \omega}},
\end{gathered}
$$

where $A^{i j \omega}=\sum_{k=1}^{n} \alpha_{k}^{i j \omega}$ and $T^{i j \omega}=\sum_{k=1}^{n} h_{k}^{i j \omega}$.

A shortcoming of this approach is that the size of the conditioning set grows according to the product of the cardinalities of the sets $\mathcal{D}, \mathcal{A}$, and $\Omega$. However, by using the concept of mixtures of Markov chains, ${ }^{(30)}$ one can linearly control the size of the conditioning set by writing

$$
\begin{aligned}
p_{D}\left(a \mid d_{i}, a_{j}, \omega\right)=w_{1} p_{D}\left(a \mid d_{i}\right) & +w_{2} p_{D}\left(a \mid a_{j}\right) \\
& +w_{3} p_{D}(a \mid \omega) .
\end{aligned}
$$

In order to make inference about the transition probabilities and weights, one can use Gibbs sampling, as described in Ref. 29. Note that the inference made on the weights may be used to check the influence of various elements $d_{i}, a_{j}$, or $\omega$ over the decisions made by Apollo, through the posterior distributions $w_{i} \mid$ data, $i=1,2,3$. This approach extends to the case in which Apollo can recall a larger number of previous games.

This Bayesian analysis is related to learning and the fictitious play approach in games. ${ }^{(31)}$ If all opponents play in this way, then under certain conditions, they converge to a Nash equilibrium, if they interact for a sufficiently long time.

As an example of a nonstrategic opponent, suppose the Attacker, Apollo, is deciding whether to attempt to plant a bomb on a train or on an a bus. Paté-Cornell and Guikema suggest ${ }^{(32)}$ that one model for his decision making is that he chooses the attack with probability proportional to his normalized utility; that is, if the utility from a train attack is twice his utility from a bus attack, he chooses the train with probability $2 / 3$ and the bus with probability $1 / 3$. There are other models for nonstrategic 
decision making, and Cox speaks ${ }^{(33)}$ about the limitations of some of these. But the probabilityproportional-to-utility model won the Best Paper award from the Military Operations Research Society, and we suppose that Daphne, the Defender, believes this is how Apollo makes his decision.

Daphne does not know the precise utilities that Apollo has, and thus does know the normalized ratio that determines the probability that he attacks the train or the bus. But, as a Bayesian, she has subjective beliefs about these. Suppose she expresses these as a Beta $(\alpha, \beta)$ distribution on $P$, the probability that he attacks the train.

Daphne can now make the decision-analytic choice that maximizes her expected utility. She believes that an attack on the train will succeed with probability $\pi_{T}^{1}$ if she does not invest in additional security, and will succeed with probability $\pi_{T}^{2}$ if she does invest in additional security, where $\pi_{T}^{2}<\pi_{T}^{1}$. The amount of investment required is $d_{T}$, and her loss from a successful attack is a known value $L_{T}$. (It would be straightforward to allow $L_{T}$ to be a random variable, reflecting the fact that the numbers of people killed and injured in an attack are uncertain, but that complicates the discussion and, in some sense, is secondary, since many of the societal costs from a successful attack do not not depend upon the body count, but rather upon the fact that an attack was actually successful.)

Similarly, Daphne believes that an attack upon a bus will have success probability $\pi_{B}^{1}$ if she does not increase bus security, and success probability $\pi_{B}^{2}$ if she does. Her cost for increasing bus security is $d_{B}$, and her loss from a successful attack in a bus is a known value $L_{B}$.

With this framework, Daphne can now calculate the expected cost (which we take to be her loss, or the negative of utility) from each of her possible choices: do nothing, improve train security only, or improve both. Specifically, since the expected value of the $\operatorname{Beta}(\alpha, \beta)$ distribution is $\alpha /(\alpha+\beta)$, then the expected loss from doing nothing is:

$$
\frac{\alpha}{\alpha+\beta} \pi_{T}^{1} L_{T}+\frac{\beta}{\alpha+\beta} \pi_{B}^{1} L_{B}
$$

the expected loss from protecting only the train is:

$$
\frac{\alpha}{\alpha+\beta} \pi_{T}^{2} L_{T}+\frac{\beta}{\alpha+\beta} \pi_{B}^{1} L_{B}+d_{T},
$$

the expected loss from protecting only the bus is:

$$
\frac{\alpha}{\alpha+\beta} \pi_{T}^{1} L_{T}+\frac{\beta}{\alpha+\beta} \pi_{B}^{2} L_{B}+d_{B},
$$

and the expected loss from protecting both is:

$$
\frac{\alpha}{\alpha+\beta} \pi_{T}^{2} L_{T}+\frac{\beta}{\alpha+\beta} \pi_{B}^{2} L_{B}+d_{T}+d_{B} .
$$

All of these quantities are known to Daphne, and she will choose the one that minimizes her expected loss.

There are, of course, other kinds of nonstrategic thinking. For example, Apollo may choose, with probability 1 , the attack that has greatest utility for him if successful, or greatest expected utility if he takes account of the possibility of failure. Daphne does not know which attack he prefers, but she has a distribution over the probability that he favors the train attack to the bus attack, and this leads to precisely the same form of calculation that we have described (and it is identical if her distribution is Beta $(\alpha, \beta))$. The structure for addressing the nonstrategic opponent is to first decide what kind or kinds of decision making he or she performs, then elicit personal probabilities over the relevant unknowns, and finally use decision analysis to select the defense that maximizes expected utility.

Repeated play occurs when Daphne and Apollo are recurring opponents in similar Defend-Attack games. Over time, Daphne can learn more about Apollo's preferences and beliefs, testing various models for his decision making and risk attitude. In this circumstance, which is not unusual in adversarial situations such as suicide bombing or cyberwarfare, Daphne's subjective beliefs about Apollo's choices may become highly informed and quite precise. Similarly, a nonstrategic Apollo might adjust his play if he finds he is too often unsuccessful.

\subsection{A Nash Equilibrium Seeking Opponent}

Suppose now that Daphne believes that Apollo will compute a Nash equilibrium to select his action. This could be because she believes that he has studied game theory or he is long-memoried nonstrategic player, as in Section 3.1., and they have played many previous games. Daphne has a subjective distribution for $\left(U_{A}, P_{A}\right)$, the random variables that represent her beliefs about Apollo's utility and probability functions $\left(u_{A}, p_{A}\right)$. She also has a subjective distribution for $\left(U_{D}, P_{D}\right)$; this is what Daphne thinks Apollo 
believes are the utility and probability functions $\left(u_{D}, p_{D}\right)$ that she uses in her analysis.

Based on the probability space $(\Theta, \mathcal{F}, \mathcal{P})$ with atomic elements $\theta \in \Theta$, the random quantities ARA needs are $\left(u_{A}^{\theta}, p_{A}^{\theta}\right) \sim\left(U_{A}, P_{A}\right)$ and $\left(u_{D}^{\theta}, p_{D}^{\theta}\right) \sim$ $\left(U_{D}, P_{D}\right)$. Also, Daphne believes the expected utilities are $\left(\psi_{D}^{\theta}(d, a), \psi_{A}^{\theta}(d, a)\right)$, with, for example,

$$
\psi_{D}^{\theta}(d, a)=\int u_{D}^{\theta}(d, a, \omega) p_{D}^{\theta}(\omega \mid d, a) d \omega .
$$

For each $\theta$, she can compute the corresponding Nash equilibrium $\left(d^{N E}(\theta), a^{N E}(\theta)\right)$, which will typically be a distribution over the $d \mathrm{~s}$ and $a$ s, respectively. Then, she would calculate:

$$
p_{D}^{N E}(a)=\mathcal{P}\left(\left\{\theta: a^{N E}(\theta)=a\right\}\right) .
$$

Ultimately, she will choose the action $d_{N E}^{*}$ that solves:

$$
d_{N E}^{*}=\operatorname{argmax}_{d} \sum_{i=1}^{n} \psi_{D}\left(d, a_{i}\right) p_{D}^{N E}\left(a_{i}\right) .
$$

In the context of a Defend-Attack game, suppose Daphne believes that Apollo will either attempt to bomb a train or a bus, and she must allocate her limited resources entirely to the defense of one or the other. Additionally, she believes that Apollo is strategic, and seeks a Nash equilibrium solution, and that he has formulated the problem as a zero-sum game, so that his gains are her losses, and conversely. (Although a zero-sum game is probably not correct, in many cases it is a reasonable approximation, especially when the consequences from success or failure have large absolute magnitude for both opponents.)

In reality, it is unlikely that Daphne will have certainty that Apollo is seeking a Nash equilibrium (unless she has a trusted informant, or knows that Apollo's Ph.D. supervisor was Roger Myerson). But she may nonetheless perform a Nash equilibrium analysis, in parallel with other models for his decision making, so that she can produce a weighted distribution over his actions, where the weights correspond to her personal probabilities that he is Nash equilibrium seeking, or is one of several kinds of nonstrategic decisionmaker, or uses any of several other strategies discussed in this article. This weighted distribution may then be used when she calculates her expected utility.

Whatever her basis, the Nash equilibrium solution concept requires that Daphne express her epistemic uncertainty about Apollo's payoff matrix. For this game, write the payoff matrix as:

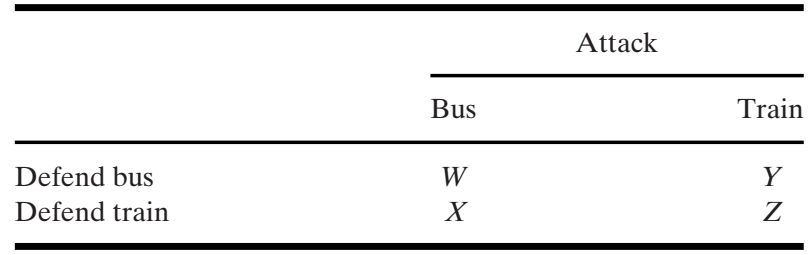

where Daphne's payoffs are the negative of Apollo's payoffs, since this is a zero-sum game. If Daphne knew the $(W, X, Y, Z)$ that Apollo held, she could solve the game and discover the action Apollo would choose, enabling her to make the best response.

But those values are generally not common knowledge. The ARA perspective implies that Daphne should treat them as random variables, so Daphne has a joint distribution over $(W, X, Y, Z)$. This joint distribution might be based upon past choices made by Apollo, or upon human intelligence, or even simple intuition. Principled elicitation of a joint distribution is not easy, ${ }^{(34)}$ but we assume that Daphne has done this, and that her personal probability for the payoffs in the table has joint density function $f(w, x, y, z)$.

One might proceed by finding the expected values of $W, X, Y$, and $Z$, and then substituting these into the payoff matrix and solving the corresponding game. But that approach is logically flawed-the operations of taking expectations and finding the minimax solution do not commute. The expected value of the minimax solution is not, in general, the minimax solution to the expected game.

Instead, Daphne must calculate her probability $p^{*}$ that Apollo will attack the bus, with $1-p^{*}$ the chance that he chooses the train. The $p^{*}$ is the weighted average of the probabilities of a bus attack over all possible values of $(w, x, y, z)$, or:

$$
\begin{array}{r}
p^{*}=\iiint \int \\
\mathbb{P}[\text { bus attack } \mid w, x, y, z] \\
\times f(w, x, y, z) d w d x d y d z .
\end{array}
$$

To solve the integral, Daphne must consider the 24 possible orderings of $W, X, Y$, and $Z$, from least to largest. These determine 24 disjoint regions of $\mathbb{R}^{4}$, denoted by $R_{1}, \ldots, R_{24}$. (This analysis ignores the possibility of ties; for continuous joint densities, this simplification does not affect the conclusion.)

If a region contains a value that is simultaneously a row minimum and a column maximum, then it has a saddlepoint. And if the table contains a saddlepoint, then Daphne will choose that row and Apollo will 
choose that column, since neither can unilaterally improve the outcome through a different choice. In a two-by-two game, there can be at most one saddlepoint. The row-column pair with $W$ is a saddlepoint if $X>W>Y$. Other possible saddlepoints are $X$, if $Z>X>W$; or $Y$, if $W>Y>Z$; or $Z$, if $Y>Z>X$. When $W$ or $X$ is a saddlepoint, the minimax theorem shows that Apollo is certain to attack the bus. If $Y$ or $Z$ are saddlepoints, she knows he will attack the train.

Saddlepoint solutions are pure strategies; that is, there is no random selection of an action. In contrast, if the table has no saddlepoint, then the minimax solution is for each player to select an action at random, where the probabilities are determined by the values $(W, X, Y, Z)$; these are called mixed strategies. For simple two-by-two games, there is a closedform expression for these probabilities. Specifically, if $W>Z>X>Y$, the game has no saddlepoint but simple algebra shows Apollo will maximize his expected gain by choosing to attack the bus with probability $p=(Z-X) /(W+Z-X-Y) .{ }^{(35)}$ The seven other orderings that do not have saddlepoints lead to analogous solutions.

By finding the corresponding pure or mixed strategy solutions in each of the 24 regions of integration, Daphne can calculate $p^{*}$, her belief about the probability that Apollo will attack the bus. In principle, this solution takes full account of all her uncertainties about the payoff table.

Daphne now uses self-elicitation to find her beliefs about her payoffs for each attack-defend combination. Importantly, her beliefs about these payoffs may be very different from those she thinks Apollo imputes to her. We denote these payoffs by $\mu_{i j}$, as follows:

\begin{tabular}{llr}
\hline & \multicolumn{2}{c}{ Attack } \\
\cline { 2 - 3 } & Bus & Train \\
\hline Defend bus & $\mu_{11}$ & $\mu_{12}$ \\
Defend train & $\mu_{21}$ & $\mu_{22}$ \\
\hline
\end{tabular}

Daphne's expected loss from defending the bus is $p^{*} \mu_{11}+\left(1-p^{*}\right) \mu_{12}$ and her expected loss from defending the train is $p^{*} \mu_{21}+\left(1-p^{*}\right) \mu_{22}$. She makes her calculation, and then chooses the action that minimizes her expected loss.

\subsection{A Level- $k$ Thinking Opponent}

When Apollo's rationality entails level- $k$ thinking, ${ }^{(36,37)}$ Daphne knows that Apollo selects his action based upon a chain of reasoning of the form "I know that she knows that I know ...," which will go $k$ levels deep, depending on how sophisticated she believes Apollo to be. Thus, if Daphne is nonstrategic, then she is a level-0 thinker and chooses randomly. If she chooses her action by assuming that Apollo is nonstrategic, then she is a level-1 thinker, and so forth. In this situation, Daphne will maximize her expected utility by reasoning one level further in the chain than Apollo.

Concretely, Daphne needs to solve Equation (1), so that her optimal decision is:

$$
d^{*}=\arg \max _{d} \sum_{i=1}^{n} \psi_{D}\left(d, a_{i}\right) p_{D}\left(a_{i}\right) .
$$

In thinking about $p_{D}(a)$, she considers the problem faced by Apollo and assumes he is an expected utility maximizer, so that his decision can be predicted by solving:

$$
\begin{aligned}
a^{*}= & \arg \max _{a} \sum_{d} \psi_{A}(d, a) p_{A}(d) \\
= & \arg \max _{a} \sum_{d}\left[\int u_{A}(d, a, \omega)\right. \\
& \left.\times p_{A}(\omega \mid d, a) d \omega\right] p_{A}(d) .
\end{aligned}
$$

Daphne does not know the elements $\left(u_{A}, p_{A}\right.$ $\left.(\cdot \mid \cdot), p_{A}\right)$ required to solve Equation (2). As a Bayesian, she expresses her uncertainty about these through random utilities and probabilities $\left(U_{A}, P_{A}(\cdot \mid \cdot), P_{A}\right)$. Substituting these in Equation (2), she obtains a predictive distribution for the random action that Apollo will choose through:

$$
\begin{aligned}
A^{*}=\arg \max _{a} \sum_{d}\left[\int\right. & U_{A}(d, a, \omega) \\
& \left.\times P_{A}(\omega \mid d, a) d \omega\right] P_{A}(d),
\end{aligned}
$$

which provides the $p_{D}(a)=\mathbb{P}\left(A^{*}=a\right)$ required in Equation (1). Here, the distribution of $A^{*}$ may be estimated through Monte Carlo simulation by sampling from the random utilities and probabilities and computing (and accumulating) the corresponding optimal alternatives as follows: 


$$
\text { Fork }=1, \ldots, \mathrm{N} \text {, repeat }
$$

Sample $\left(u_{A}^{k}, p_{A}^{k}(\cdot \mid \cdot), p_{A}^{k}\right) \sim\left(U_{A}, P_{A}(\cdot \mid \cdot), P_{A}\right)$

Compute $a_{k}^{*}=\operatorname{argmax}_{a} \sum_{d}\left[\int u_{A}^{k}(d, a, \omega)\right.$

$$
\left.\times p_{A}^{k}(\omega \mid d, a) d \omega\right] p_{A}^{k}(d)
$$

Approximate $p_{D}\left(a_{i}\right)$ by

$\operatorname{card}\left\{1 \leq k \leq N: a_{k}^{*}=a_{i}\right\} / N$,

for $i=1, \ldots, n$

In the above triplet $\left(U_{A}, P_{A}(\cdot \mid \cdot), P_{A}\right)$, the first two elements are relatively easy for Daphne to elicit, since they represent what Daphne believes are Apollo's utility function and beliefs about the outcome of the game, conditional on their decisions. For example, $P_{A}(\omega \mid d, a)$ could be centered around her own $p_{D}(\omega \mid d, a)$ with some additional uncertainty. As far as $U_{A}$ is concerned, typically she will have information about the interests of Apollo, which she would aggregate with a weighted measurable utility function. Using the relative risk aversion concept, ${ }^{(38)}$ she could model the risk attitude of Apollo that determines the functional form of his utility function. Finally, her uncertainty would be reflected by distributions over the weights and the risk coefficient.

In contrast, the third element, $P_{A}(d)$, often requires higher-level strategic thinking. She must model what Apollo thinks is Daphne's decision analysis. Thus, if Daphne supposes that Apollo is a level1 thinker and he is modeling her as an expected utility maximizer, then from his perspective Daphne is modeled as solving the optimization problem in Equation (1) where the required $\left(u_{D}, p_{D}(\cdot \mid \cdot), p_{D}\right)$ are unknown to him and, therefore, must be represented through random utilities and probabilities $\left(U_{D}, P_{D}(\cdot \mid \cdot), P_{D}\right)$. This allows Apollo to elicit his predictive probability distribution over her possible actions through:

$$
\begin{aligned}
p_{A}(D=d)= & \mathbb{P}\left(\operatorname { a r g } \operatorname { m a x } _ { x \in \mathcal { D } } \sum _ { a } \left[\int U_{D}(x, a, \omega)\right.\right. \\
& \left.\left.\times P_{D}(\omega \mid x, a) d \omega\right] P_{D}(a)=d\right),
\end{aligned}
$$

which he needs to solve Equation (2).

Now, Daphne's uncertainty about Apollo's distribution $F_{D}$ for $\left(U_{D}, P_{D}(\cdot \mid \cdot), P_{D}\right)$ can be modeled through $F_{D} \sim \mathcal{F}_{D}$, her probabilistic beliefs about the distributions $F_{D}$ used by Apollo to solve her decision problem, and compute his $p_{A}(d)$. This gives the $P_{A}(d)$ required in Equation (3) through:

$$
\begin{aligned}
P_{A}(D=d)= & \mathbb{P}\left(\operatorname { a r g } \operatorname { m a x } _ { x \in \mathcal { D } } \sum _ { a } \left[\int U_{D}(x, a, \omega)\right.\right. \\
& \left.\left.\times P_{D}(\omega \mid x, a) d \omega\right] P_{D}(a)=d\right)
\end{aligned}
$$

with $\left(U_{D}, P_{D}(\cdot \mid \cdot), P_{D}\right) \sim F_{D}$ and now $F_{D} \sim \mathcal{F}_{D}$.
Once Daphne obtains $P_{A}(d)$, she plugs it into Equation (3) to obtain the $p_{D}(a)$ required in Equation (1), thus making Daphne a level-2 thinker. This level- $k$ thinking process would continue, to the level that Daphne deems necessary, as in the following loop, which constitutes a hierarchy of nested decision models:

Repeat from $i=2$ :

$$
\begin{aligned}
P_{D}^{i}\left(A^{i}=a\right)= & \mathbb{P}\left(\underset { x \in \mathcal { A } } { \operatorname { a r g } \operatorname { m a x } _ { d \in \mathcal { D } } } \left[\int U_{A}^{i}(d, x, \omega)\right.\right. \\
& \left.\left.\times P_{A}^{i}(\omega \mid d, x) d \omega\right] P_{A}^{i}\left(D^{i}=d\right)=a\right) \\
\text { with } \quad & \left(U_{A}^{i}, P_{A}^{i}(\cdot \mid \cdot), P_{A}^{i}\right) \sim F_{A}^{i} \\
& F_{A}^{i} \sim \mathcal{F}_{A}^{i} \\
P_{A}^{i}\left(D^{i}=d\right)= & \mathbb{P}\left(\operatorname { a r g } \operatorname { m a x } _ { x \in \mathcal { D } } \sum _ { a \in \mathcal { A } } \left[\int U_{D}^{i}(x, a, \omega)\right.\right. \\
& \left.\left.\times P_{D}^{i}(\omega \mid x, a) d \omega\right] P_{D}^{i+1}\left(A^{i+1}=a\right)=d\right) \\
& \left(U_{D}^{i}, P_{D}^{i}(\cdot \mid \cdot), P_{D}^{i+1}\right) \sim F_{D}^{i} \\
& F_{D}^{i} \sim \mathcal{F}_{D}^{i}
\end{aligned}
$$

$i=i+1$

Note that $p_{D}(a)$ and $P_{A}(d)$ defined, respectively, by Equations (3) and (5), would correspond to a step $i=1$ in the above loop. To sum up the levels of thinking in level- $k$ rationality, in terms of the notation we have used,

- A level-0 Daphne acts at random (nonstrategically).

- A level-1 Daphne chooses her alternative optimally, but assumes that Apollo acts randomly, since he is level-0, as in Section 3.1.

- A level-2 Daphne assumes that Apollo is a level-1 adversary, who assumes she is a level0 thinker. Daphne stops at $i=1$ in the hierarchy, with the elicitation of $F_{D} \sim \mathcal{F}_{D}$, which determines $P_{A}(d)$ representing her beliefs about the probability model used by Apollo to predict her action.

- A level-3 Daphne assumes that she faces a level2 adversary: Apollo's calculation assumes she is a level-1 thinker, who thinks about his decision problem. Daphne stops at $i=2$ in the hierarchy, with the elicitation of $F_{A}^{2} \sim \mathcal{F}_{A}^{2}$, which determines $P_{D}^{2}\left(A^{2}=a\right)$.

- A level-4 Daphne assumes she is facing a level3 adversary: Apollo takes strategic account of what he thinks she thinks he thinks that she 
thinks, Daphne stops at $i=2$ in the hierarchy, with the elicitation of $F_{D}^{2} \sim \mathcal{F}_{D}^{2}$, which determines $P_{A}^{2}\left(D^{2}=d\right)$.

- In general, a level- $k$ Daphne models her opponent as a level- $(k-1)$ adversary, enabling recursion as above.

There is no natural end to this regression. Daphne wants to think exactly one level more deeply than Apollo, so the termination rule depends upon how subtle she thinks Apollo is.

Rothschild et al. (39) use this framework to provide an algorithmic approach to level- $k$ thinking. Daphne first selects the value $k>1$ that she believes is the depth of Apollo's analysis. Then she places a uniform distribution over Apollo's actions and supposes that Apollo has a uniform distribution over her action space. Daphne climbs up one level at a time in the hierarchy by simulating from these distributions and solving to find the new distribution for Apollo's optimal action, and consequently inferring his corresponding new distribution over her own action set. She repeats until she reaches the selected value of $k$.

We believe it is more natural to think in terms of an alternative approach, as suggested in Refs. 1 and 4, which proceeds by climbing up in the hierarchy until Daphne finds it difficult to reason meaningfully. Indeed, at higher levels of thinking, Daphne will probably lack the information necessary to assess the distributions $\mathcal{F}_{A}^{i}$ or $\mathcal{F}_{D}^{i}$ associated with the decision analysis of $A^{i}$ or $D^{i}$, respectively. At this point, Daphne might assign a probability distribution over $A^{i}$ or $D^{i}$, without going deeper in the hierarchy, thus summarizing all remaining information she might have through the direct assessment of $P_{D}^{i}\left(A^{i}=a\right)$ or $P_{A}^{i}\left(D^{i}=d\right)$, as appropriate. At this stage, one reasonable possibility is to assign a noninformative distribution. Lee and Wolpert ${ }^{(40)}$ describe experiments in behavioral game theory suggesting that opponents rarely go further than levels $k=2$ or 3. So, in most cases, Daphne need not to go beyond $k=3$ or 4 in order to be one level deeper than Apollo.

For the running example of a Defend-Attack game, we have already covered the case in which Daphne is a level-1 thinker. Daphne models Apollo as a nonstrategic opponent, and her optimal decision is obtained through the logic described in Section 3.1.

Now suppose that Daphne does a level-2 ARA, so she assumes Apollo is a level-1 thinker. This model implies that Daphne thinks Apollo assumes that she is a level-0 thinker. Apollo must place a probability over her decision on whether to defend the bus or the train (she lacks the resources to pro- tect both). Let $q$ be his probability that she defends the bus, and let $u_{B}$ be his utility from a successful attack on the bus, and $u_{T}$ be his utility from a successful attack on the train. Apollo thinks his probability of success when attacking an undefended bus is $p_{B}^{1}$, and his probability of success when attacking a defended bus is $p_{B}^{2}$, where $p_{B}^{2}<p_{B}^{1}$. Similarly, Apollo thinks his probability of success when attacking an undefended train is $p_{T}^{1}$, and his chance of success when attacking a defended train is $p_{T}^{2}$, where $p_{T}^{2}<p_{T}^{1}$. Thus, Apollo's expected utility from a bus attack is $q p_{B}^{2} u_{B}+(1-q) p_{B}^{1} u_{B}$ and his expected utility from a train attack is $q p_{T}^{1} u_{T}+(1-q) p_{T}^{2} u_{T}$. Apollo will choose whichever attack provides greatest expected utility.

Daphne, of course, does not know $q, p_{B}^{1}, p_{B}^{2}$, $p_{T}^{1}, p_{T}^{2}, u_{B}$, or $u_{T}$. But, perhaps based on a combination of previous choices made by Apollo, military intelligence, and personal intuition, she has a joint density over $\left(Q, P_{B}^{1}, P_{B}^{2}, P_{T}^{1}, P_{T}^{2}, U_{B}, U_{T}\right)$, the random variables representing her uncertainty about Apollo's values. She now propagates her uncertainty into Apollo's calculation, so that she has a distribution for $Q P_{B}^{2} U_{B}+(1-Q) P_{B}^{1} U_{B}$, his expected utility from a bus attack, and over $Q P_{T}^{1} U_{T}+(1-Q) P_{T}^{2} U_{T}$, his expected utility from a train attack. This enables her to find her personal value for:

$$
\begin{aligned}
p= & \mathbb{P}\left[\left(Q P_{B}^{2}\right.\right. \\
& \left.+(1-Q) P_{B}^{1}\right) U_{B} \\
& \left.>\left(Q P_{T}^{1}+(1-Q) P_{T}^{2}\right) U_{T}\right],
\end{aligned}
$$

or the probability that Apollo will choose to attack the bus.

To complete her analysis, the level-2 Daphne consults her own, known, losses $L_{B}$ from a successful bus attack and $L_{T}$ from a successful train attack, and her own beliefs $\pi_{B}^{1}$ and $\pi_{B}^{2}$, the probabilities of a successful attack upon an undefended bus and a defended bus, respectively, and $\pi_{T}^{1}$ and $\pi_{T}^{2}$, the probabilities of successful attack upon an undefended and defended train, respectively. Assuming a successful defense entails no loss to Daphne, her expected utility from defending the bus is $p \pi_{B}^{2} L_{B}+$ $(1-p) \pi_{T}^{1} L_{T}+d_{B}$ and her expected utility from defending the train is $p \pi_{B}^{1} L_{B}+(1-p) \pi_{T}^{2} L_{T}+d_{T}$. She makes the choice that minimizes her expected loss.

One can proceed, so that Daphne is a level-3 thinker, performing an ARA for an Apollo who is a level-2 thinker, and so forth. The blueprint for such extension is provided in Section 3.5. 


\subsection{Mirror Equilibria Seeking Opponents}

As implied above, level- $k$ thinking can lead to an infinite regress. Classical game theory avoids this through the common knowledge assumption, which allows players to use deterministic predictive models of their opponents decisions. Another way to preclude the infinite regress is through the mirroring equilibria concept, ${ }^{(3)}$ which we formalize here.

Assume Daphne has distributions for the random quantities $\left(U_{A}, P_{A}(\cdot \mid \cdot), P_{A}(\cdot)\right)$, which describe Daphne's beliefs about Apollo's utilities and probabilities, as in Equation (3), and she also has $\left(U_{D}, P_{D}(\cdot \mid \cdot), P_{D}(\cdot)\right)$, which describe Daphne's beliefs about Apollo's beliefs regarding her own utilities and probabilities, as in Equation (4). This completes step $i=1$ in the hierarchy for level- $k$ thinking.

Suppose for a moment that Daphne has a point mass on $\theta$, in the generic probability space $(\Theta, \mathcal{F}, \mathcal{P})$ introduced previously. In this case, she believes that Apollo will solve for his optimal decision:

$$
\begin{array}{r}
a^{*}(\theta)=\arg \max _{a} \sum_{d}\left[\int u_{A}^{\theta}(d, a, \omega)\right. \\
\left.\times p_{A}^{\theta}(\omega \mid d, a) d \omega\right] p_{A}^{\theta}(d) .
\end{array}
$$

Next, by assuming nonpoint mass support $\mathcal{P}$, she deduces her predictive distribution over Apollo's choice in $\mathcal{A}$,

$$
p_{D}^{M E}(a)=\mathcal{P}\left(\left\{\theta \in \Theta: a^{*}(\theta)=a\right\}\right) .
$$

Note that this may be written as:

$$
\begin{aligned}
p_{D}^{M E}(a)= & \mathbb{P}\left(\operatorname { a r g } \operatorname { m a x } _ { x \in \mathcal { A } } \sum _ { d } \left[\int U_{A}(d, x, \omega)\right.\right. \\
& \left.\left.\times P_{A}(\omega \mid d, x) d \omega\right] P_{A}(d)=a\right) .
\end{aligned}
$$

Symmetrically, knowing $\theta$ and $p_{D}^{M E}(a)$, Daphne thinks that Apollo believes that she is trying to solve:

$$
\begin{array}{r}
d^{*}(\theta)=\arg \max _{d}\left[\sum_{d} \int u_{D}^{\theta}(d, a, \omega)\right. \\
\left.\times p_{D}^{\theta}(\omega \mid d, a) d \omega\right] p_{D}^{M E}(a),
\end{array}
$$

which yields a random optimal decision $d^{*}(\theta)$ on the underlying probability space $(\Theta, \mathcal{F}, \mathcal{P})$ with distribution:

$$
p_{A}^{M E}(d)=\mathcal{P}\left(\left\{\theta \in \Theta: d^{*}(\theta)=d\right\}\right) .
$$

We say that the distributions $p_{D}^{M E}(a)$ and $p_{A}^{M E}(d)$ are consistent and constitute a mirroring equilibrium if they jointly satisfy:

$$
\left.\begin{array}{rl}
p_{A}^{M E}(d)= & \mathcal{P}\left(\operatorname { a r g } \operatorname { m a x } _ { x } \sum _ { a } \left[\int U_{D}(x, a, \omega)\right.\right. \\
& \left.\left.\times P_{D}(\omega \mid x, a) d \omega\right] p_{D}^{M E}(a)=d\right) \\
p_{D}^{M E}(a)= & \mathcal{P}\left(\operatorname { a r g } \operatorname { m a x } _ { x } \sum _ { d } \left[\int U_{A}(d, x, \omega)\right.\right. \\
& \left.\left.\times P_{A}(\omega \mid d, x) d \omega\right] p_{A}^{M E}(d)=a\right) .
\end{array}\right\}
$$

When such a pair of consistent distributions is found, this provides Daphne with a probabilistic model to predict Apollo's actions, in which it is assumed that he uses the mirroring equilibria as the solution concept. At this point, Daphne steps out of the mirror equilibrium paradigm and uses her true utility and probability functions to select the action that maximizes her expected utility; that is,

$$
\max _{d} \sum_{i=1}^{n} \psi_{D}\left(d, a_{i}\right) p_{D}^{M E}\left(a_{i}\right),
$$

with $p_{D}^{M E}(a)$ obtained as the fixed point solution of the mirroring analysis in Equations (6). The mirroring solution concept may be viewed as a way to enforce coherence in Daphne's probability judgments.

The existence of fixed points solutions to the mirroring distribution formulation in Equations (6) is a complex question and only partial solutions are generally available. The problem is closely related to the existence of Bayes Nash equilibria solutions. The most complete theory exists in the context of twoperson asymmetric auctions. ${ }^{(41,42)}$

For the two-person simultaneous DefendAttack game, the mirroring equilibrium solution is almost equivalent to the Bayes Nash equilibrium solution. That solution assumes there is common knowledge about the payoff matrix, the probabilities of success conditional on different actions, and the losses and utilities to the Defender and Attacker, respectively. This common knowledge is probabilistic, which is expressed as a distribution over the types of the players. ${ }^{(11)}$

In this framework, both Apollo and Daphne know the decision problem that each is solving. Daphne thinks the probability that Apollo chooses to attack the bus is:

$$
\begin{array}{r}
p=\mathbb{P}\left[q P_{B}^{2} U_{B}+(1-q) P_{B}^{1} U_{B}>\right. \\
\left.q P_{T}^{1} U_{T}+(1-q) P_{T}^{2} U_{T}\right]
\end{array}
$$


and, symmetrically, Apollo thinks the probability that Daphne chooses to defend the bus is:

$$
\begin{array}{r}
q=\mathbb{P}\left[p \Pi_{B}^{2} L_{B}+(1-p) \Pi_{T}^{1} L_{T}+D_{B}<\right. \\
\left.p \Pi_{B}^{1} L_{B}+(1-p) \Pi_{T}^{2} L_{T}+D_{T}\right],
\end{array}
$$

where there is common knowledge about the joint distribution of all capitalized random variables, and each opponent knows their own type. For example, the values of the losses $L_{B}$ and $L_{T}$ are known to Daphne, and she knows the distribution that Apollo has for those values. With this machinery, Harsanyi ${ }^{(43-45)}$ was able to show that equilibria $\left(p^{*}, q^{*}\right)$ exist such that neither opponent can unilaterally improve his or her outcome by employing a different (mixed) strategy for selecting his or her action.

From the ARA perspective, one can drop the common knowledge assumption. Instead, all the random variables are owned by, say, Daphne, who thinks, as a Bayesian, that Apollo holds these beliefs about her probabilities and losses, and that he also believes she has the corresponding distributions over his values. The solution is a little different at the last step, since Daphne solves the equilibrium problem to obtain her estimate of the probability that Apollo will attack the bus, and then makes the choice that maximizes her true expected utility, using probabilities and losses that may be very different from the ones imputed to Apollo. But the ARA solution diverges strongly from the Bayes Nash equilibrium solution when there are more than two opponents, since it becomes possible to describe what Daphne thinks about what Apollo thinks about Bob, and what Daphne thinks about Bob, in practical ways that are inconsistent with common knowledge.

\subsection{A Cognitive Comparison}

We compare now the four previous models for opponent rationality, and the standard gametheoretic approach, in terms of the cognitive load that the analysis imposes upon Daphne. Table I summarizes the elements that must be assessed according to the hierarchy in level- $k$ thinking introduced in Section 3.3.

Row 0 corresponds to the utilities and beliefs of Daphne and Apollo, as perceived by themselves. The elements in row 1 all correspond to random utilities and probabilities perceived by Daphne, including those she believes are Apollo's utilities and probabilities, subscripted by $A$, and those she believes are Apollo's beliefs about her own utilities and probabil- ities, subscripted by $D$. Row 2 includes what she believes Apollo believes are her beliefs about Apollo's utilities and probabilities, subscripted by $A$, and so forth.

To summarize the cognitive demands of the various approaches, we shall refer to element $(i, j)$ in the table as the cognitive element in row $i$ and column $j$. All the approaches we have discussed require the elements $(0,1)$ and $(0,3)$. Beyond that:

- The standard game-theoretic approach requires also elements $(0,2)$, and $(0,5)$, and these must be common knowledge. This limits the scope in realistic applications.

- The nonstrategic opponent model requires also $(0,4)$, but uses no specific strategic principles in assessing it; as sketched in Section 3.1., Daphne needs only data and/or expert opinion.

- As sketched in Section 3.2., the Nash equilibrium opponent model requires elements $(1,1)$, $(1,3),(1,2)$, and $(1,5)$, which are used to calculate $(0,4)$.

- The level- $k$ opponent model requires, as sketched in Section 3.3., these elements:

- $(1,2),(1,5)$, and $(1,6)$, which are used to produce $(0,4)$, or,

- $(1,1),(1,3)$, and $(1,4)$, which are used to produce $(1,6)$, and this in turn, with $(1,2)$ and $(1,5)$ produces $(0,4)$, or,

- additional layers are needed to handle $k>3$.

- The mirror equilibrium opponent model requires elements $(1,2),(1,5)$ and $(1,1),(1,3)$, and uses the consistency condition to produce $(0,4)$, as sketched in Section 3.4.

Any of the solution concepts discussed in this article (and others) might be the right one to use. If Daphne thinks Apollo is a level-2 thinker, then she should perform a level-3 analysis. If she believes he seeks a Bayes Nash equilibrium, then her best response requires that she models him as such. And, of course, if she is uncertain about which type of strategist he is, then she needs to do both analyses and combine her inferences, as discussed in Section 4.

However, the depth of thinking shown in this table is often helpful to Daphne in assessing her beliefs about the solution concept Apollo might use. If she thinks Apollo is not very smart or not very determined, this increases the chance that he is nonstrategic, or perhaps just a level-1 thinker, since these impose relatively lighter cognitive burdens. If she 
Table I. Information that Daphne Must Assess to Obtain ARA Solutions When Apollo Employs One of the Solution Concepts Discussed

\begin{tabular}{ccccccc}
\hline & 1 & 2 & 3 & 4 & 5 & 6 \\
\hline 0 & $u_{D}$ & $u_{A}$ & $p_{D}(\cdot \mid d, a)$ & $p_{D}(a)$ & $p_{A}(\cdot \mid d, a)$ & $p_{A}(d)$ \\
1 & $U_{D}$ & $U_{A}$ & $P_{D}(\cdot \mid d, a)$ & $P_{D}(a)$ & $\left.P_{A}(d), a\right)$ & $P_{A}^{2}(d)$ \\
2 & $U_{D}^{2}$ & $U_{A}^{2}$ & $P_{D}^{2}(\cdot \mid d, a)$ & $P_{D}^{2}(a)$ & $\vdots$ \\
$\vdots$ & $\vdots$ & $\vdots$ & $\vdots$ & $\vdots$ & $\vdots$ \\
\hline
\end{tabular}

believes Apollo thinks there is common knowledge, then she thinks it is more likely that he will seek a (Bayes) Nash equilibrium solution. And if she thinks Apollo is tenacious and well resourced, then he might undertake the more complex analyses required for higher rungs of level- $k$ thinking.

\subsection{Prospect Maximizing Opponents}

One possible criticism of the opponent models presented so far is that they assume that both players essentially seek to maximize expected utility. The assumption is arguably reasonable for Daphne-ARA is designed to support her decision making, and can prescribe that perspective for her. But it is more tenuous for Apollo, and there is much evidence that humans often make choices that do not maximize expected utility. ${ }^{(46)}$ In the context of counterterrorism, both terrorism psychology ${ }^{(47)}$ and logistics ${ }^{(48)}$ suggest that adversaries tend to invest their attack resources in order to produce the best outcomes in a certain sense. A well-reported example is the corporate-like organization of piracy in Somalia; ${ }^{(5)}$ referred to as cutthroat capitalism. ${ }^{(49)}$ Thus, attackers optimize, but they might not be optimizing expected utility.

An alternative descriptive model of decision making under uncertainty is prospect theory; ${ }^{(50)}$ here one optimizes some criterion other than expected utility. To illustrate how the expected utility solutions may be extended to other optimization criteria, suppose that Apollo's choice is modeled as the solution of:

$$
\begin{aligned}
a^{*}= & \arg \max _{a}\left[\sum_{d} \int v_{A}(d, a, \omega)\right. \\
& \left.\times \pi_{A}\left(p_{A}(\omega \mid d, a)\right) d \omega\right] \pi_{A}\left(p_{A}(d)\right),
\end{aligned}
$$

where $v_{A}$ is a value function and $\pi_{A}$ is a weighting function over Apollo's probabilities $p_{A}$, as defined in Ref. 51. Note the formal similarity with Equation (2).
Daphne does not know these probability distributions and value and weighting functions. She must elicit personal distributions over them, which we model through $\left(V_{A}, \Pi_{A}, P_{A}(\cdot \mid \cdot), P_{A}\right)$. By propagating the uncertainty quantified in these distributions, she induces a probability distribution over Apollo's action space, as in Equation (3), through:

$$
\begin{aligned}
A^{*}= & \arg \max _{a}\left[\sum_{d} \int V_{A}(d, a, \omega)\right. \\
& \left.\times \Pi_{A}\left(P_{A}(\omega \mid d, a)\right) d \omega\right] \Pi_{A}\left(P_{A}(d)\right) .
\end{aligned}
$$

This distribution is the $p_{D}^{P T}(a)=\mathbb{P}\left(A^{*}=a\right)$ required for her to maximize her expected utility in Equation (1). Also as before, Daphne could examine a hierarchy of nested decision analysis, in the more complex level- $k$ framework, in order to ultimately elicit $p_{D}^{P T}(a)$.

\section{COMBINING OPPONENT MODELS}

The five procedures that we have discussed correspond to different opponent models. These lead to different adversarial forecasting models and, consequently, potentially different optimal actions for Daphne. But in many situations she will not know which model among these possibilities (and others) correctly describes Apollo's behavior. However, as a Bayesian, she will have a subjective probability about the relevance of each model she considers. These subjective probabilities allow her to combine her predictions through a Bayesian mixture model. ${ }^{(13,14)}$

Let $p\left(M_{i}\right)$ be the probability that Daphne has for each of the $k$ opponent models that she thinks might describe Apollo, with $\sum_{i=1}^{k} p\left(M_{i}\right)=1$ and $p\left(M_{i}\right) \geq 0, i=1, \ldots, k$. Let $p_{D}^{i}(a)$ be the probability distribution induced by each opponent model over Apollo's action set, through the kind of analysis described in Section 3. Daphne then combines all these distributions into a single distribution $p_{D}(a)=$ $\sum_{i=1}^{k} p\left(M_{i}\right) p_{D}^{i}(a)$, a weighted average for which the 
weights are her beliefs about the type of reasoning Apollo will use. She should then solve:

$$
\begin{aligned}
d^{*} & =\max _{d} \sum_{a} \psi_{D}(d, a)\left(\sum_{i=1}^{k} p\left(M_{i}\right) p_{D}^{i}(a)\right) \\
& =\max _{d} \sum_{i=1}^{k} p\left(M_{i}\right)\left[\sum_{a} \psi_{D}(d, a) p_{D}^{i}(a)\right]
\end{aligned}
$$

to determine her best possible choice.

For example, with Defend-Attack games, suppose Daphne thinks that with probability 0.25 Apollo is nonstrategic and with probability 0.75 , he is a level-1 thinker. Under the assumptions made previously for these two cases, Daphne would find her distribution for Apollo's choice under both models for his strategic analysis. Let $p_{D}^{1}(a)$ be her probability distribution for his choice when he is nonstrategic, and $p_{D}^{2}(a)$ be her distribution for his choice when he is a level-1 thinker. Then Daphne would form the mixture distribution $p_{D}(a)=0.25 p_{D}^{1}(a)+$ $0.75 p_{D}^{2}(a)$ and maximize her expected utility against this distribution over his choices to find whether she should defend the bus or the train.

When there is repeated play, Daphne can learn what kind of rationality Apollo employs, and thus improve her model for Apollo's decision making. By appropriately embedding these as parametric models, she can build in a model selection strategy based on $p\left(M_{i} \mid\right.$ data $)$, as data about games accumulate. At a given time she could decide to use in her mixture only opponent models that have sufficiently high posterior probability, or, if one model emerges as a clear winner, she could use it solely. But, as argued in Ref. 52, this underestimates her uncertainty, which may be disastrous in certain applications, such as national security. ${ }^{(53)}$ This Bayesian model selection strategy may be also seen as a model validation approach. As data accumulate in repeated play, $p\left(M_{i} \mid\right.$ data $)$ will go to 1 if $M_{i}$ reflects the solution concept that Apollo actually uses, and otherwise it goes to 0 . Thus, $p\left(M_{i} \mid\right.$ data $)$ may be viewed as a measure of how valid Daphne believes model $M_{i}$ to be. See Ref. 54 for additional discussion of this perspective.

\section{DISCUSSION}

We have provided a description of a family of opponent models for ARA, in the context of discrete, simultaneous two-person games, which we have illustrated with a running example of a Defend-Attack game. The ideas extend easily to continuous games. The extension to other forms of interaction, such as sequential defend-attack or sequential defendattack-defend, ${ }^{(4,55)}$ may become complicated, but the approach is clear. When extending the methods to multiple adversaries, one needs to consider whether to allow for the possibility that opponents coordinate their decisions. Beyond that, there are also technical issues, such as determining when mirroring equilibria exist, or determining whether it is necessary to climb higher in the level- $k$ hierarchy, taking into account value-of-information concepts. Additionally, although we discussed some aspects of learning from repeated play, much more could be done.

There is no simple solution for a serious strategic analysis. But this is exactly the conclusion one should expect. Daphne's success depends critically upon the accuracy of the information that she has about her opponent, and, in particular, it is often sensitive to what she believes Apollo believes about her.

In general, Daphne will not have precise knowledge about what kind of rationality (or solution concept) Apollo is using to select his action. But, at the cost of additional computation, Daphne can use a mixture model to combine the results from different opponent models, incorporating her model uncertainty, and then use this distribution to choose the action probabilities, and ultimately determine what solution concept is most likely being used. From this perspective, ARA provides a flexible and attractive framework for making strategic decisions.

\section{ACKNOWLEDGMENTS}

The work of DRI was supported by the AXA-ICMAT Chair in Adversarial Risk Analysis, the MINECO projects MTM2011-28983-C3-1 and MTM2014-56949-C3-1-R, the FP7 SECONOMICS grant 285223, and the ISCH COST Action IS1304 on Expert Judgement.

\section{REFERENCES}

1. Rios Insua D, Rios J, Banks D. Adversarial risk analysis. Journal of the American Statistical Association, 2009; 104:841-854.

2. Wang S, Banks D. Network routing for insurgency: An adversarial risk analysis framework. Naval Research Logistics, 2011; 58:595-607.

3. Banks D, Petralia F, Wang S. Adversarial risk analysis: Borel games. Applied Stochastic Models in Business and Industry, 2011; 27:72-86.

4. Rios J, Rios Insua D. Adversarial risk analysis for counterterrorism modeling. Risk Analysis, 2012; 32:894-915.

5. Sevillano JC, Rios Insua D, Rios J. Adversarial risk analysis: The Somali pirates case. Decision Analysis, 2012; 9:86-95.

6. Razuri J, Esteban PG, Rios Insua D. An advervsarial risk analysis model for an autonomous imperfect decision agent. Pp. 163-187 in Guy TV, Karny M, Wolpert D (eds). Decision 
Making and Imperfection. Berlin: Springer Verlag, 2012 .

7. Kadane J, Larkey B. Subjective probability and the theory of games. Management Science, 1982; 28:113-120.

8. Raiffa $\mathrm{H}$. The Art and Science of Negotiation. Harvard University Press, Cambridge, MA, 1982.

9. Raiffa H, Richardson J, Metcalfe D. Negotiation Analysis: The Science and Art of Collaborative Decision. Cambridge, MA: Harvard University Press, 2002.

10. Harsanyi J. Comment: Subjective probability and the theory of games: Comments on Kadane and Larkey's paper. Management Science, 1982; 28:120-124.

11. Myerson R. Game Theory: Analysis of Conflict. Cambridge, MA: Harvard University Press, 1991.

12. Savage LJ. Foundations of Statistics. New York: John Wiley, 1954.

13. Hoeting J, Madigan D, Raftery A, Volinsky C. Bayesian model averaging: A tutorial. Statistical Science, 1999; 14:382417.

14. Clyde M, George E. Model uncertainty. Statistical Science, 2004; 19:81-94.

15. Chipman HA, George EI, McCulloch RE. The Practical Implementation of Bayesian Model Selection, IMS Lecture Notes-Monograph Series, Vol. 38, Model Selection, 2001, pp. 65-116. Available at: http://www-stat.wharton.upenn.edu edgeorge/Research_papers/ims.pdf.

16. Koller D, Milch B. Multi-agent influence diagrams for representing and solving games. Games and Economic Behavior, 2003; 45:181-221.

17. Hausken K, Bier V, Zhuang J. (2008) Defending against terrorism, natural disaster, and all hazards. Pp. 65-97 in Bier V, Azaiez N (eds). Game Theoretic Risk Analysis of Security Threats. New York: Springer, 2009.

18. Bier V, Azaiez MN. Game Theoretic Risk Analysis of Security Threats. New York: Springer, 2009.

19. Zhuang J, Bier V. Reasons for secrecy and deception in homeland-security resource allocation. Risk Analysis, 2010; 30:1737-1743.

20. Parnell G, Smith C, Moxley D. Intelligent adversary risk analysis: A bioterrorism risk management model. Risk Analysis, 2010; 30:32-48.

21. Banks D, Rios J, Ríos Insua D. Adversarial Risk Analysis. New York: Taylor \& Francis, 2015.

22. Merrick J, Parnell GS. A comparative analysis of PRA and intelligent adversary methods for counterterrorism risk management. Risk Analysis, 2011; 31:1488-1510.

23. Gibbons R. A Primer in Game Theory. Harlow: Pearson Education Ltd. 1992.

24. Nash J. Non-cooperative games. Annals of Mathematics, 1951; 54(2):286-295.

25. Rothkopf M. Decision analysis: The right tool for auctions. Decision Analysis, 2007; 4:167-172.

26. Lippman S, McCardle K. Embedded Nash bargaining: Risk aversion and impatience. Decision Analysis, 2012; 9:3140.

27. Chung KL. A Course in Probability Theory, 2nd ed. (Chapter 1). New York: Academic Press, 1974.

28. French S, Rios Insua D. Statistical Decision Theory. London: Arnold, 2000.

29. Rios Insua D, Ruggeri F, Wiper M. Bayesian Analysis of Stochastic Process Models. Chichester, UK: Wiley, 2012.

30. Raftery AE. A model for high-order Markov chains. Royal Statistical Society Series B, 1985; 47:528-539.

31. Menache I, Ozdaglar A. Network Games. Morgan Claypool Pub, 2011. Available at: http://www.morganclaypool.com/.

32. Paté-Cornell E, Guikema S. Probabilistic modeling of terrorist threats: A systems analysis approach to setting priorities among countermeasures. Military Operations Research, 2002; 7:5-23.

33. Cox Jr LA . What's wrong with risk matrices. Risk Analysis, 2008; 28:497-512.

34. O'Hagan A, Buck C, Daneshkhah A, Eiser JR, Garthwaite $\mathrm{PH}$, Jenkinson DJ, Oakley JE, and Rakow T. Uncertain Judgements: Eliciting Experts' Probabilities. Hoboken, NJ: Wiley, 2006.

35. Rapoport A. Two Person Game Theory: The Essential Ideas (Chapter 7). Ann Arbor, MI: University of Michigan Press, 1970.

36. Stahl D, Wilson P. Experimental evidence on players' models of other players. Journal of Economic Behavior and Organization, 1994; 25:309-327.

37. Stahl D, Wilson P. On players' models of other players: Theory and experimental evidence. Games and Economic Behavior, 1995; 10:218-254.

38. Dyer JS, Sarin RK. Measurable multiattribute value functions. Operations Research, 1979; 27(4):810-822.

39. Rothschild C, McLay L, Guikema S. Adversarial risk analysis with incomplete information: A level- $k$ approach. Risk Analysis, 2012; 32:1219-1231.

40. Lee R, Wolpert D. Game theoretic modeling of pilot behavior during mid-air encounters. Pp. 75-111 in Karny TV, Guy M, Wolpert DH (eds). Decision Making with Imperfect Decision Makers. Heidelberg: Springer Verlag, 2012.

41. Lebrun B. Existence of an equlibrium in first-price Auctions. Economic Theory, 1996; 7:421-443.

42. Lebrun B. First price auctions in the asymmetric $N$ bidder case. International Economic Review, 1999; 40:125142.

43. Harsanyi J. Games with incomplete information played by bayesian players. Part I. The basic model. Management Science, 1967; 14:159-182.

44. Harsanyi J. Games with incomplete information played by bayesian players. Part II. Bayesian equilibrium points. Management Science, 1967; 14:320-334.

45. Harsanyi J. Games with incomplete information played by bayesian players. Part III. The basic probability distribution of the game. Management Science, 1968; 14;486-502.

46. Camerer C. Behavioral Game Theory. Princeton, NJ: Princeton University Press, 2003.

47. English R. Terrorism. Oxford Univ. Press, 2009.

48. Brown G, Carlyle WM, Salmeron J, Wood K. Defending critical infrastructure. Interfaces, 2006; 36:530-544.

49. Carney S. Cutthroat capitalism: An economic analysis of the Somali pirate business model. Wired, 2009. Available at: http://archive.wired.com/politics/security/magazine/1707/ff_somali_pirates.

50. Wakker P. Prospect Theory: For Risk and Ambiguity. Cambridge University Press, 2010.

51. Kahneman D, Tversky A. Prospect theory: An analysis of decision under risk. Econometrica, 1979; 47(2):263-291.

52. Draper D. Assessment and propagation of model uncertainty. Journal of the Royal Statistical Society, Series B, 1995; 57:4597.

53. Sunstein CR. Worst-Case Scenarios. Harvard University Press, 2007.

54. Berger J, Rios Insua D. Recent developments in Bayesian inference with applications in hydrology. Pp. 56-80 in Parent E, Hubert P, Miquel J, Bobee B (eds). Statistical and Bayesian Methods in Hydrology. Paris: UNESCO Press, 1998.

55. Shan X, Zhuang J. Hybrid defensive resource allocation of partially strategic attackers in a sequential defender-attacker game. European Journal of Operational Research, 2013; 228:262-272. 